\title{
On the correlation of spatial wind speed and solar irradiance variability above the North Sea
}

\author{
Anna Rieke Mehrens and Lueder von Bremen \\ ForWind, Center for Wind Energy Research, Department of Physics, Carl von Ossietzky University Oldenburg, \\ Oldenburg, Germany \\ Correspondence to: Anna Rieke Mehrens (anna.mehrens@ forwind.de)
}

Received: 15 January 2016 - Revised: 1 April 2016 - Accepted: 4 April 2016 - Published: 12 April 2016

\begin{abstract}
Mesoscale wind fluctuations on a time scale of tens of minutes to several hours lead to high wind power fluctuations. Enhanced mesoscale wind variability emerges during cold air outbreaks and resulting cellular convection. The study investigates spatial wind and solar variability and their correlation during cellular convection. Cellular convection leads to simultaneous high solar and wind variability, but the highest solar or wind variability occurs due to other meteorological phenomena.
\end{abstract}

\section{Introduction}

Mesoscale wind fields on a time scale of tens of minutes to several hours can be very variable above the North Sea. An example how mesoscale wind fluctuations are transformed into wind power fluctuations is shown in Fig. 1. Around the 8 February 2009, the wind speed shows recurring wind speed changes. These wind fluctuations are in a wind speed range, where the power curve is steep. Consequently the wind fluctuations are transformed to very large power fluctuations.

Vincent and Hahmann (2011), Heggem et al. (1998) and others showed that a typical weather situation, which enhances mesoscale wind variability, are cold air outbreaks on the rear of low pressure systems. When the cold air is advected over warm water, cellular clouds develop. It is also possible to simulate these fluctuations in mesoscale models (Vincent et al., 2012; Vincent, 2010).

The fluctuations in the example time series in Fig. 1 occur during such a cold air outbreak with cellular convection, which is visible in the satellite image in Fig. 2.

If mesoscale fluctuation occurs during cellular convection, the question arises if it is possible to estimate the variability of the wind by the variability of the solar irradiance field? The solar field can be derived from satellite measurements.

Under the assumption that temporal mesoscale wind fluctuations occur because of a spatial inhomogeneous wind field which is advected to a measurement point, the relation between the spatial variability of the wind field and the spatial variability of the solar field is analysed in this study, in particular during cellular convection cases.

\section{Wind data}

Due to the lack of mesoscale spatial measurements of the chosen site, simulated wind speed data of the Weather Research \& Forecasting Model (WRF) are used. The WRF dataset has a spatial resolution of $1.75 \mathrm{~km}$ and a time resolution of $10 \mathrm{~min}$. The simulation uses MERRA as initial and boundary conditions (Rienecker et al., 2011) and the NCEP OISST dataset (Reynolds et al., 2002) for the daily update of the Sea surface Temperature. For the planetary Boundary Layer subgrid parametrisation the YSU scheme and for the Cumulus parametrisation the Kain-Fritsch (new Eta) scheme was used. The model runs without any reinitialisations and is driven by the lateral boundary conditions to obtain a continuous time series. The analysed area is a part of the third domain with a size of $105 \mathrm{~km} \times 105 \mathrm{~km}$ (Fig. 3) and data in a height of $54 \mathrm{~m}$ are used. Figure 4 compares the power spectral density of the WRF wind speed with the offshore measurements at Fino1 to test if the model set up is capable to simulate mesoscale wind fluctuations. Although the measurement and the simulated time series are not at the exact same position, it can be seen that the model underestimates the power spectral density, especially at the mesoscale. 
(a)

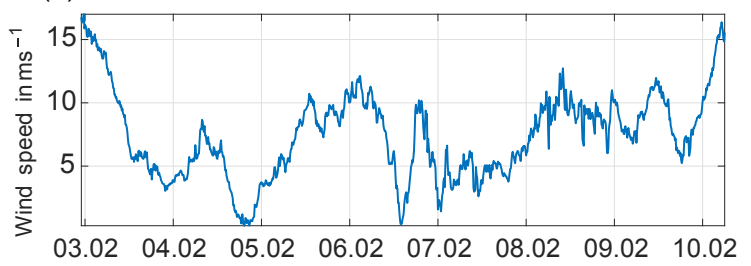

(b)

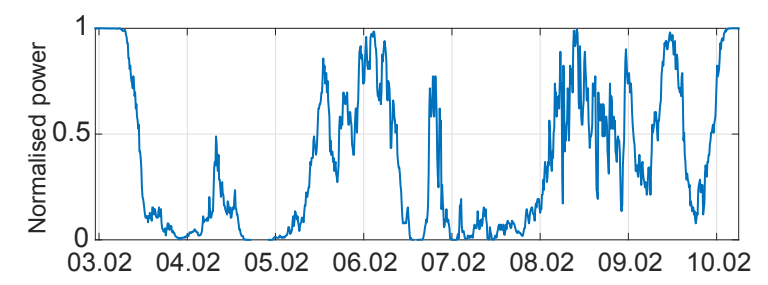

Figure 1. Example wind speed time series of the offshore met mast Fino 1 (a) and resulting wind power time series by using a power curve of an offshore turbine (b). The power is normalized with the maximum power.

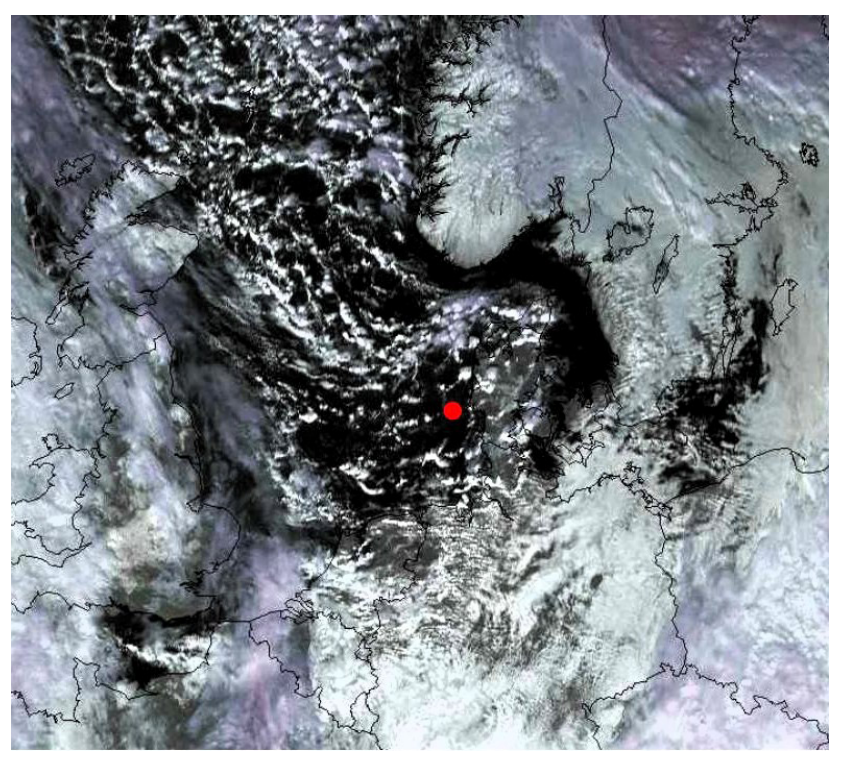

Figure 2. Satellite image of the 8 February 2009 12:00 UTC by Meteosat. The red dot marks the study domain above the North Sea (Fig. 3).

\section{Solar data}

To calculate the spatial solar variability, global horizontal irradiance (ghi) data, which are derived by the Heliosat method, are used (Hammer, 2014; Hammer et al., 2003). The data have a spatial resolution of $2.2 \mathrm{~km}$ and a time resolution of $15 \mathrm{~min}$. For the comparison with the WRF wind field, the same $1.75 \mathrm{~km}$ grid is used. Consequently the nearest solar grid point value is chosen. This may lead to a reduced spatial variability of the solar field than the wind field, because 685 points out of 3600 points have the same value due to

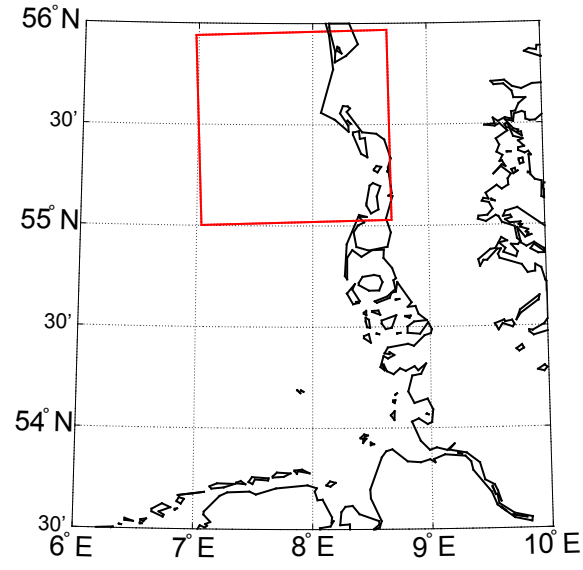

Figure 3. Analysed study domain to calculate the spatial variability of the wind and solar field.

the lower resolution of the satellite data than the wind simulation. Both datasets cover the same study domain and the whole year of 2009 .

\section{Methodology}

To obtain mesoscale fluctuations only, the time series are filtered with a Fast Fourier Transformation filter. This filter removes the energy of all frequencies with a cycle duration of $6 \mathrm{~h}$ and longer.

Figure 4 shows the power spectral density of the original and the filtered time series of one solar irradiance and one wind speed grid point in the study domain. The comparison of the original and the filtered power spectral density demonstrates that the filtered time series have the same spectral power density like the original time series, but all scales, with greater cycle durations than the mesoscale, are removed.

This procedure provides a fluctuation time series for wind and solar. The result for the wind speed is shown in Fig. 5. Figure 5a shows the original time series of the wind speed at one grid point of the WRF simulation and a wind speed measurement at the nacelle of one wind turbine, which is located in a wind park in the study domain. The fluctuation time series for the simulation and the measurement after the filtering procedure are shown in Fig. 5b. Because of the filter, the larger time scales are removed and the wind speed fluctuates around zero. The mesoscale fluctuations at the 8 February, are clearly visible. It is also visible that the fluctuations are shifted in the WRF simulation data towards the next days. A likely reason is the model set up without reinitialisation. Thus, the simulation and the measurements have a phase shift. This filter is applied on all grid point time series in the wind and solar field. The result is a spatial wind and solar field which contains mesoscale fluctuations only.

To measure the variability of the solar and wind fields, the longitudinal and lateral increments between the grid points 


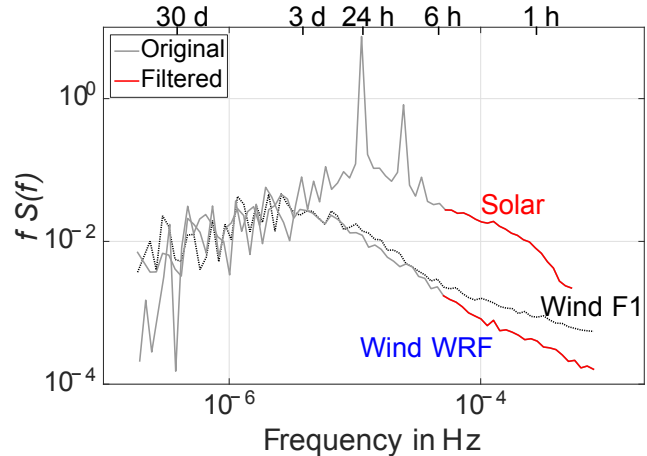

Figure 4. Power spectral density of the WRF wind speed and the solar irradiance for the original time series in black and in red for a time series where all cycle durations higher than $6 \mathrm{~h}$ are removed with a Fast Fourier Transform filter. The dashed black line shows the Power spectral density for the wind speed measurements for the same year in $50 \mathrm{~m}$ height at Fino 1.

are added (spatial increment sum). Because of the phase shift in the measurements and the WRF simulation a daily mean is calculated. The daily mean increment sum is then normalized by the average of the year to get dimensionless results.

To compare the spatial variability of wind and solar for days with cellular convection, a measure is needed to select these days. Currently no general method exists to detect cellular convection above the North Sea. It was found that stability measures like the Richardson number are not sufficient. Thus, days in 2009 are chosen based on satellite images of Meteosat, which clearly show cellular convection (Fig. 2). It is cross checked if these fluctuations are resolved in the WRF simulation. Based on this procedure three days in 2009 are found.

Figure 6 illustrates the fluctuation time series and the spatial field during a cold air outbreak. The filtered wind speed and solar time series (Fig. 6a) show strong fluctuations. The spatial field of the filtered wind time series shows structures which move with the wind direction through the chosen area (Fig. 6b). The solar radiation fluctuation field is also very heterogeneous due to clouds (Fig. 6c).

\section{Results and discussion}

The comparison of the mesoscale spatial fluctuation of wind and solar shows that the solar variability has a stronger pronounced yearly cycle (Fig. 7). Although the yearly cycle was filtered, the monthly mean value of the spatial fluctuation measure reaches a maximum in the summer months of 2009 and is minimal in the winter months. In contrast to this, the spatial wind fluctuation measure has a smaller maximum in winter and a higher minimum in summer. Thus, the monthly mean of the spatial mesoscale variability of wind and solar are opposed.
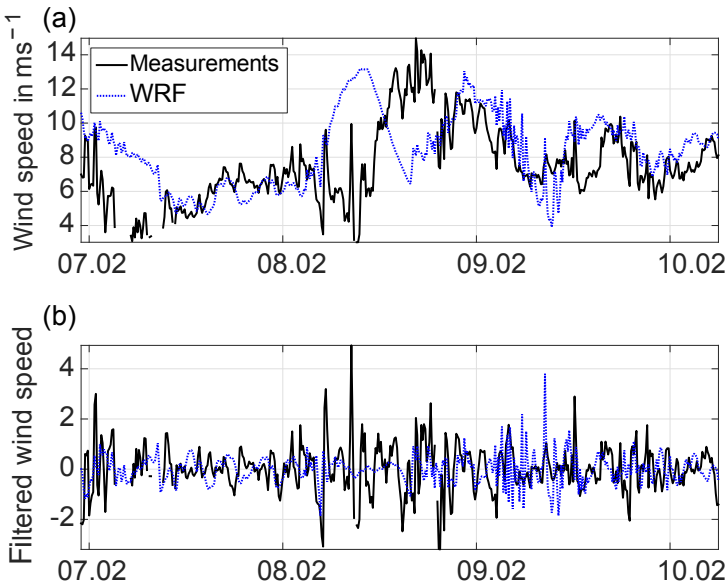

Figure 5. Original wind speed time series of one grid point of the WRF simulation (a) and fluctuation time series after filtering (b).

Figure 8 shows the relation between the daily fluctuation measures for wind and solar. The three days with investigated cellular convection are marked in green. These days do not yield to maximal spatial solar and wind variability. Thus, there have to be other meteorological phenomena which yield either to higher spatial solar or wind fluctuations. All of the days with the highest mean values for the solar variability are in summer. The days with the highest values for the wind variability are in winter. The figure also demonstrates that there is no linear relation which would allow to expect mesoscale wind fluctuations, when there is a very heterogeneous solar field.

However, Fig. 8 demonstrates that the example days with prevailing open cellular convection have comparable variability measure values for solar and wind.

Beside of the investigation of the influence of open cellular convection on the relation between the solar and wind variability, the Richardson number, a classification of the daily circulation (Climatic Research Unit, 2016; Jones et al., 2013), and the wind direction are examined. Days that were selected based on this indicators did not show a relation between wind and solar variability.

The resolution of the original solar data grid is lower than the resolution of the wind grid, but the results show that the daily solar variability is not lower than the wind variability.

\section{Conclusions}

This study demonstrates that cellular convection leads to simultaneous high spatial solar and wind fluctuations, but the highest fluctuations occur due to other weather phenomena.

Due to the fact that the WRF simulation does not resolve all mesoscale fluctuation cases and the unavailability of a method to detect cellular convection, the sample size was reduced to only three test cases. 
(a)

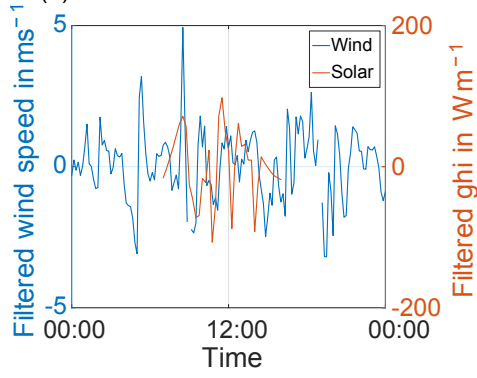

(b)

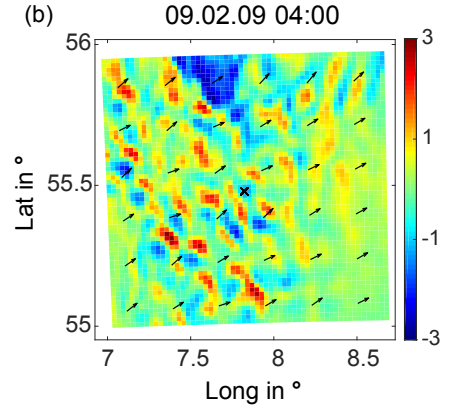

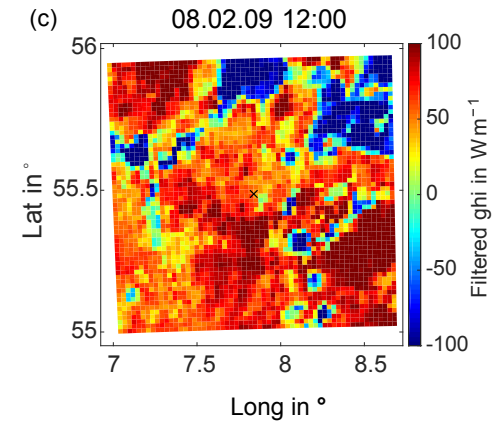

Figure 6. Fluctuation time series and spatial field for a cellular convection case. Panel (a) shows a filtered measurement at a wind turbine whose position is marked in (b) and the filtered solar time series for the measurement point, marked in (c). Panel (b) shows the WRF fluctuation wind field and (c) the solar fluctuation field. Because of the phase shift between the measurements and the WRF simulation, (b) shows a later time step.

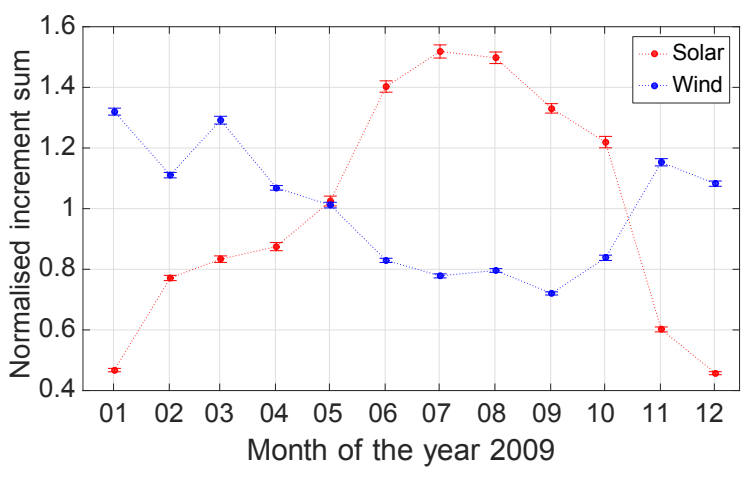

Figure 7. Monthly mean of the 10 min spatial mesoscale wind and solar variability. The errorbars show the standard error.

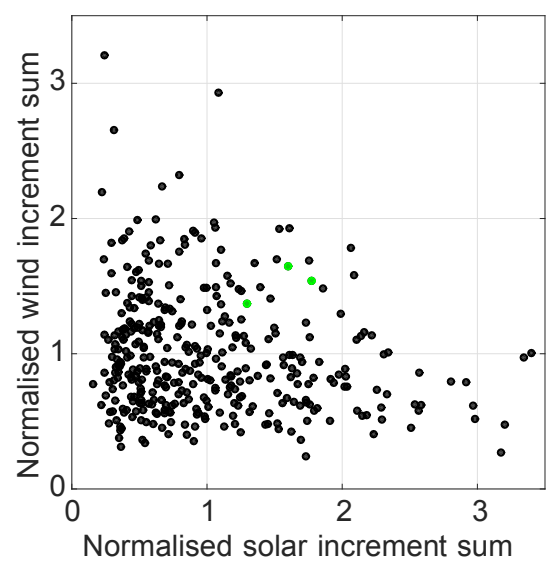

Figure 8. Daily spatial wind and solar variability for all days in the year 2009. The days with cellular convection are marked in green.

The spatial analysis of mesoscale wind fields is strongly hampered by the unavailability of measurements with a good spatial and temporal resolution. Mesoscale models may close this gap, as they are capable to resolve mesoscale fluctuations. However, depending on the simulation set up and ini- tialisation, a phase shift between measurement and simulation may occur. In future studies we want to use Lidar scans, to investigate the spatial variability of wind fields. But these measurements have a smaller study domain than the simulation.

Acknowledgements. The work presented in this study is funded by the ministry of science and culture of Lower Saxony within the PhD Program System Integration of Renewable Energies and the project ventus efficiens (ZN3024, MWK Hannover). We thank Axel Kemper (University of Oldenburg, EnMetSol) for providing the solar data, Francisco Javier Santos Alamillos (University of Reading) for providing the WRF wind data and the Federal Maritime and Hydrographic Agency (BSH) for providing the Fino 1 measurements. We thank the reviewers for their constructive reviews.

Edited by: S.-E. Gryning

Reviewed by: two anonymous referees

\section{References}

Climatic Research Unit: Reanalysis based LWTs, Grid centred on the N. Sea, available at: https://crudata.uea.ac.uk/cru/data/lwt/, last access: 30 March 2016.

Hammer, A.: Documentation - EnMetSOL, Satellite Data - Available Regions at Oldenburg University, Tech. rep., University of Oldenburg, available at: http://www.uni-oldenburg.de/fileadmin/user_upload/physik/ ag/ehf/enmet/download/enmetsol-documentation.pdf (last access: 15 January 2016), 2014.

Hammer, A., Heinemann, D., Hoyer, C., Kuhlemann, R., Lorenz, E., Muller, R., and Beyer, H.: Solar energy assessment using remote sensing technologies, Remote Sens. Environ., 86, 423-432, doi:10.1016/S0034-4257(03)00083-X, 2003.

Heggem, T., Lende, R., and Løvseth, J.: Analysis of Long Time Series of Coastal Wind, J. Atmos. Sci., 55, 2907-2917, doi:10.1175/1520-0469(1998)055<2907:AOLTSO>2.0.CO;2, 1998. 
Jones, P. D., Harpham, C., and Briffa, K. R.: Lamb weather types derived from reanalysis products, Int. J. Climatol., 33, 11291139, doi:10.1002/joc.3498, 2013.

Reynolds, R. W., Rayner, N. A., Smith, T. M., Stokes, D. C., and Wang, W.: An Improved In Situ and Satellite SST Analysis for Climate, J. Climate, 15, 1609-1625, doi:10.1175/15200442(2002)015<1609:AIISAS>2.0.CO;2, 2002.

Rienecker, M. M., Suarez, M. J., Gelaro, R., Todling, R., Bacmeister, J., Liu, E., Bosilovich, M. G., Schubert, S. D., Takacs, L., Kim, G.-K., Bloom, S., Chen, J., Collins, D., Conaty, A., da Silva, A., Gu, W., Joiner, J., Koster, R. D., Lucchesi, R., Molod, A., Owens, T., Pawson, S., Pegion, P., Redder, C. R., Reichle, R., Robertson, F. R., Ruddick, A. G., Sienkiewicz, M., and Woollen, J.: MERRA: NASA's Modern-Era Retrospective Analysis for Research and Applications, J. Climate, 24, 3624-3648, doi:10.1175/JCLI-D-11-00015.1, 2011.
Vincent, C.: Mesoscale wind fluctuations over Danish waters, $\mathrm{PhD}$ thesis, Technical University of Denmark, Ris $\emptyset$, National Laboratory for Sustainable Energy, 2010.

Vincent, C. and Hahmann, A.: Hour-scale wind fluctuations over the North Sea, pp. 190-193, European Wind Energy Conference and Exhibition 2011, Scientific Proceedings, 2011.

Vincent, C. L., Hahmann, A. N., and Kelly, M. C.: Idealized Mesoscale Model Simulations of Open Cellular Convection Over the Sea, Bound.-Lay. Meteorol., 142, 103-121, doi:10.1007/s10546-011-9664-7, 2012. 